\title{
Studies on Grounding Technologies for Power System in China
}

\author{
Jinliang $\mathrm{He}^{*}$ Non-member \\ Rong Zeng* Non-member
}

\begin{abstract}
The problems related to grounding systems of substations and power plants include geological structure analysis of substation site, the precise design of grounding system, the technology to decrease grounding resistance of substation in high soil resistivity area, corrosion diagnosis of grounding system, precise measurement of grounding resistance of substation, and impulse characteristics of grounding devices. Up to present, these six aspects related to grounding technology of power system have been widely studied, and achieved satisfied results in China. These achievements were systematically introduced in this paper.
\end{abstract}

Keywords: power system, grounding technology, corrosion diagnosis, grounding resistance, substation

\section{Introduction}

The grounding system of substation should ensure the safe and reliable operation of power system, and guarantee human being's safety in the situation of grounding fault in power system. In China, there are a lot of faults caused by bad grounding systems in power system, which destroyed the power apparatus and control systems. Up to present, the main problems related to grounding systems of substations and power plants have been widely studied, and achieved satisfied results in China. These achievements were systematically introduced in this paper.

\section{Analysis on Soil Structure Parameters for Grounding System Design}

Reasonable and accurate estimation of the soil resistivity is fundamental for the grounding system design. Ordinarily, the apparent soil resistivity data are measured by Wenner four-electrode configuration, and then the soil structure parameters are derived by numerical method.

A fast algorithm is presented to invert the structure parameters of the horizontal multi-layer soil. The procedure is divided into two independent stages. First, Fredholm equation of the first kind with respect to the apparent resistivity is solved by the technology of decay spectrum to reduce computation time greatly. Second, the structure parameter of soil is determined by the generalized Newton-Kantorovich method, which is more robust and less noise sensitive because of using the generalized inverse algorithm to solve the nonlinear equation group ${ }^{(1)}$.

The measured apparent soil resistivity curve from Zibo $220 \mathrm{kV}$ Qianyu Substation is shown in Fig. 1, and the soil structure can be analyzed as four layers, the resistivity and thickness of the top layer are $19 \Omega \cdot \mathrm{m}$ and $0.32 \mathrm{~m}$, those of the second layer are $42 \Omega \cdot \mathrm{m}$ and $2.4 \mathrm{~m}$, those of the third layer are $522 \Omega \cdot \mathrm{m}$ and $4.62 \mathrm{~m}$, the resistivity of the bottom layer is $573 \Omega \cdot \mathrm{m}^{(2)}$.

\footnotetext{
* The State Key Lab of Power Systems, Dept. of Electrical Engineering,

Tsinghua University

Beijing 100084, China
}

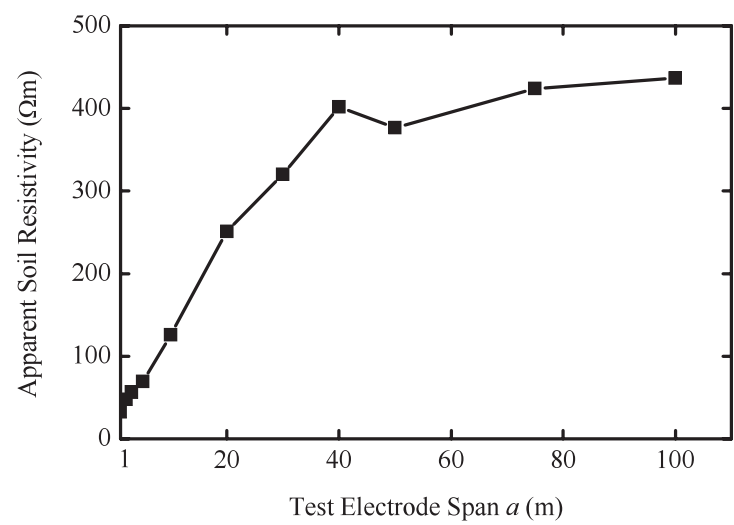

Fig. 1. The apparent soil resistivity curve of Zibo 220 kV Qianyu Substation

\section{Grounding System Design}

3.1 Numerical Analysis of Grounding System The numerical calculation of grounding system based on multilayer soil model is the fundament of grounding system design. Ordinarily, numerical calculation methods proposed in literatures were based on constant-current field theory. When a dc or ac current is injected into a grounding system, the potential in an arbitrary point satisfies the Lapalace's equation, and all conductors of grounding system are subdivided into segments, the integral problem is simplified as a summation problem. Based on these, the leakage current distribution in the grounding system can be solved, then the potential in an arbitrary point can be obtained. The key is how to calculate the Green's function. The coefficients of Green's function are straightforwardly sampled in an iterative way in terms of the equation group satisfying the pertinent boundary value problem, then the closed-form expression of multilayered soil Green's function can be given by the vector matrix pencil technology ${ }^{(2)}$.

The developed analysis software had been applied in the design of 40 grounding systems. The errors between the numerical analysis and field test are all controlled less than $10 \%$. 


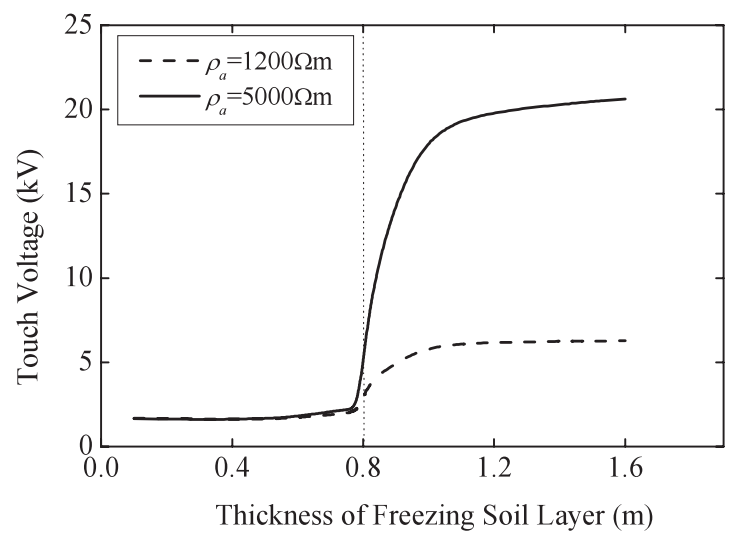

Fig. 2. The influence of the thickness of the freezing soil layer on the touch voltage of the ground surface $\left(\rho_{a}\right.$ is the resistivity of frozen soil layer)

3.2 Influence of Seasonal Frozen Soil Layer In different seasons, the resistivity of the surface soil layer would be changed, which would affect the safety of grounding systems, and the grounding resistance, step and touch voltages would move to the safe side, or to the hazard side, it is our concerned question ${ }^{(3)}$. As shown in Fig. 2, when the thickness of the freezing soil layer exceeds the burial depth of the grounding system, the touch voltage sharply increases.

The frozen season would change the soil model, the homogeneous soil would become a two-layer soil in frozen season. The final design scheme of the grounding system should be determined synthetically from two phases. Firstly the grounding system is arranged according to the optimum design in normal soil model and its safety is checked in seasonal frozen soil model, secondly it is arranged according to the optimum design in frozen soil model and its safety is checked in normal soil model ${ }^{(4)}$.

\section{New Method to Decrease Grounding Resis- tance}

High grounding resistance would affect the safe operation of power system. Two different methods are used to decrease grounding resistance of substation.

The first is the explosive grounding technique (EGT) ${ }^{(5)}$. First, several vertical holes are drilled, and then appropriate explosive agents are introduced into the holes, at last lowresistivity material (LRM) is filled into these formed cracks, as illustrated in Fig. 3. This network acts like a virtual extension of the grounding system to deeper soils, resulting in lower ground resistances. The LRM is usually an inorganic material with a resistivity less than $5 \Omega \cdot \mathrm{m}$. The explosion course is carefully planned, and the region close to the surface remains intact. This method has been verified very effectively in China, and now it has been applied in about 30 grounding projects.

The second method is the deep ground well method ${ }^{(6)}$, which uses deep wells to change the moving directions of groundwater in the soil surrounding the grounding electrodes to increase the humidity of the soil surrounding the grounding electrode, which will decrease the soil resistivity neighboring the grounding substation and consequently decrease the grounding resistance of the grounding electrode. As

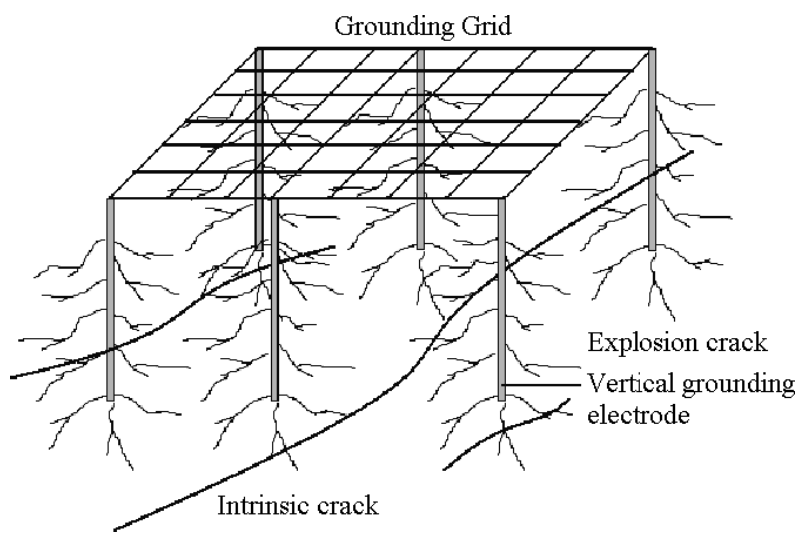

Fig. 3. The cubic grounding system formed by explosive grounding technique

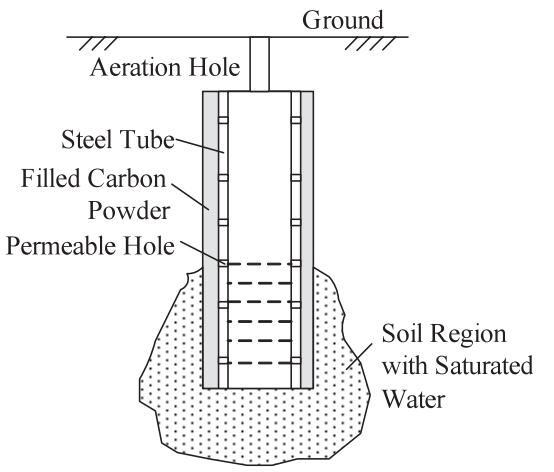

Fig. 4. Schematic diagram of deep-ground-well

shown in Fig. 4, the deep ground wells use steel tubes with water percolation apertures as the electrodes. The diameter of the equivalent low-resistivity region numerically analyzed from field experiments is from $0.57 \mathrm{~m}$ to $4.13 \mathrm{~m}$, and the average diameter is $2.93 \mathrm{~m}^{(6)}$.

\section{Corrosion Diagnosis System of Substation Grounding Grid}

In China, the grounding grid is made of steel. When the steel conductors are corroded, it is a potential problem and very dangerous to the power system, since it is buried under ground. A grounding grid can be handled as a circuit network, every conductor of the grounding grid is a branch, and every cross point of these conductors is a node. It is possible to measure port resistances among any two down-lead conductors connected with the grounding grid. If the relationship between port resistance and conductor resistance is given, conductor resistance can be computed from port resistances through mathematical analysis. Comparing these results with the initial values that they are designed to be, the accurate current corrosion status of all grounding conductors under ground can be known ${ }^{(7)}$.

A novel corrosion diagnosis system is proposed as shown in Fig. $5^{(9)}$, by which corrosion status of grounding system can be diagnosed from measured port resistances among the touchable down-lead conductors connected to the grounding grid.

The analyzed corrosion degrees of every grounding conductors of a substation by the developed corrosion diagnosis system are shown in Fig. 6. 


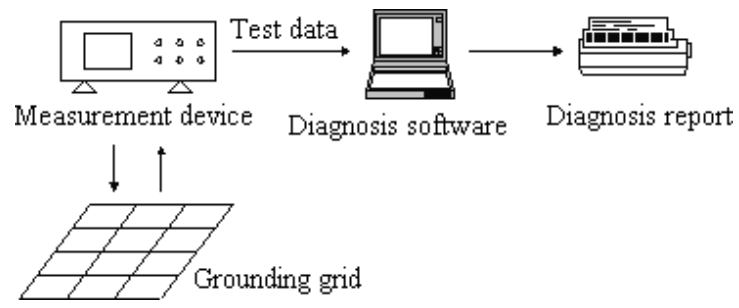

Fig. 5. The structure diagram of corrosion diagnosis system

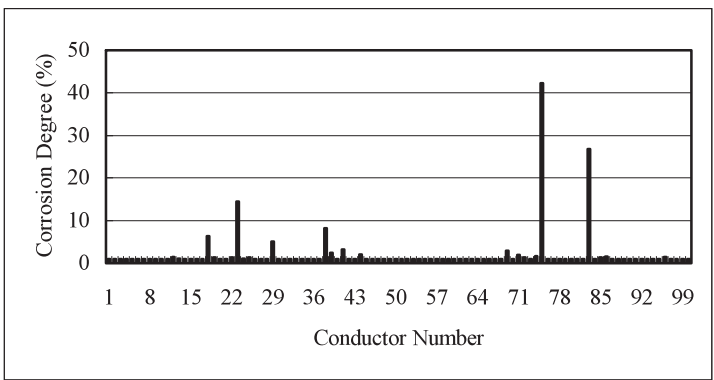

Fig. 6. The corrosion degree of every grounding conductors of a substation analyzed by the corrosion diagnosis system

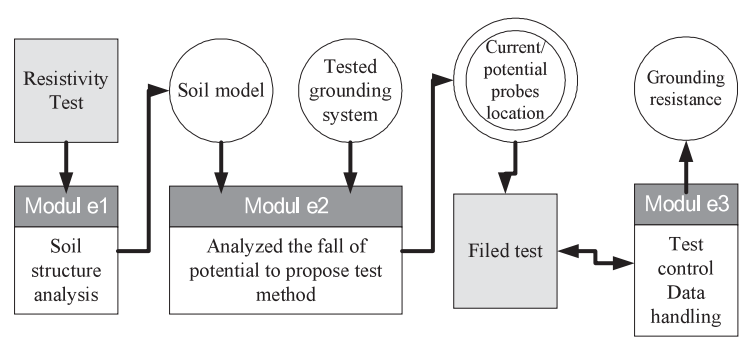

Fig. 7. The overall structure of intelligent grounding resistance measuring system

\section{Test Technique of Grounding Resistance of Substation}

An intelligent measuring system of grounding resistance based on the fall-of-potential method is developed as shown in Fig. $7^{(8)}$. A frequency-variable power source is used for grounding resistance measurement, which can automatically expel the influence of interference current. The test system can provide arrangement rule of measuring electrodes for grounding resistance test circuit ${ }^{(9)}$. The influence of overhead ground wire on grounding impedance of substation can be handled according to the method introduced in Ref. (10).

\section{Impulse Performances of Grounding Devices and Grounding Grids}

7.1 Simulating Experiment of Grounding Devices

Reducing grounding resistance of a transmission tower grounding device is an important method to improve performance of transmission lines against lightning. Impulse experiments using grounding device models were systematically performed to analyze the influence of different factors on the impulse characteristics of grounding devices. Formulae to

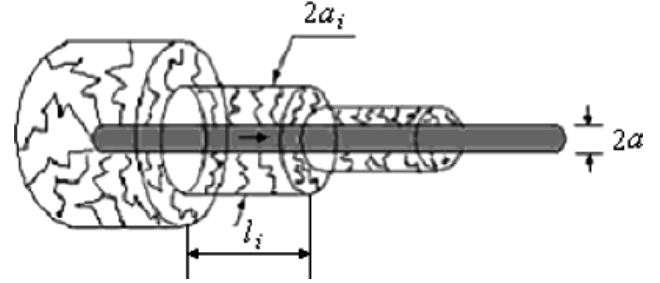

Fig. 8. Modeling of equivalent radii for each segment

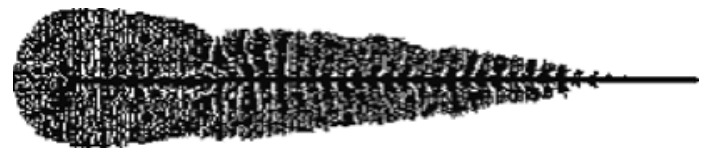

Fig. 9. Discharge pattern produced by fractal simulation

calculate impulse coefficients of different grounding devices were proposed in Ref. (11).

7.2 Impulse Performance Analysis of Grounding Device Considering Soil Ionization When the electric field strength surrounding the grounding conductor exceeds the critical value of soil breakdown electrical field strength, the soil around the grounding electrode would be breakdown. The nonlinear loss transmission line model proposed in Ref. (12) is shown in Fig. 8, the conductor is represented by a set of cylindrical zones to simulate the soil ionization phenomena, $a_{i}$ in Fig. 13 is the equivalent radius of the $i$ th segment, which is time-variable when an impulse current is injected into the grounding electrode; and $a$ is the radius of the metal conductor.

On the other hand, a stochastic probability model of discharge initiation and propagation processes around electrode in soil under lightning current is presented, which is based on fractal theory ${ }^{(13)}$. Fig. 9 shows a discharge pattern produced by fractal simulation.

If the grounding electrode in soil is very long, it can not be used efficiently, because the grounding electrode has "effective length". And the conception of the impulse effective length of grounding electrodes under impulse current is explained in Ref. (13). For $40 \mathrm{kA}$ lightning current, from experimental and analyzed results, the effective length for one arm of center-injecting single horizontal electrode was concluded as

$$
l_{e}=7.683(\rho \tau)^{0.379} / I_{M}^{0.097}
$$

And the effective length for one arm of center-injecting 4arm star counterpoise wires can be calculated by:

$$
l_{e}=8.963(\rho \tau)^{0.379} / I_{M}^{0.097}
$$

7.3 Impulse Performance Analysis of Grounding Grid Considering Soil Ionization A circuit model with lumped time-variable parameter is proposed in Ref. (14) to calculate the transient characteristic of a grounding grid under lightning current, which especially considers the dynamic and non-linear effect of soil ionization around grounding conductors and the mutual impedance among any two grounding conductors. The influences of soil parameters, location of feed point, and shape of the lightning impulse current on the transient characteristic of grounding grid are discussed. The 
effective impulse range of grounding grid is analyzed, which will be helpful for technical staff to design grounding grid in the protection against lightning ${ }^{(14)}$.

\section{Acknowledgment}

This work was supported by the National Natural Science Foundations of China under Grants 50407002 and 50425721.

(Manuscript received Oct. 25, 2004)

\section{References}

( 1 ) J. Zou, J.L. He, R. Zeng, W.M. Sun, G. Yu, and S.M. Chen: "Two-stage algorithm for inverting structure parameters of the horizontal multi-layer soil", IEEE Trans. Magnetics, Vol.40, No.2, pp.11361-1139 (2004)

( 2 ) J. Zou, R. Zeng, J.L. He, J. Guo, Y.Q. Gao, and S.M. Chen: "Numerical green's function of a point current source in horizontal multi-layer soils by utilizing the vector matrix pencil technique", IEEE Trans. Magnetics, Vol.40, No.2, pp.730-733 (2004)

( 3 ) J. He, R. Zeng, Y. Gao, Y. Tu, W. Sun, J. Zou, and Z. Guan: "Seasonal influences on safety of substation grounding system", IEEE Trans. Power Delivery, Vol.18, No.3, pp.788-795 (2003-7)

( 4 ) J. He, Y. Gao, R. Zeng, W. Sun, J. Zou, and Z. Guan: "Optimal design of grounding system considering the influence of seasonal frozen soil layer," IEEE Trans. Power Delivery, Vol.20, No.1, pp.107-115 (2005-1)

( 5 ) Q. Meng, J. He, F.P. Dawalibi, and J. Ma: "A new method to decrease ground resistances of substation grounding systems in high resistivity regions", IEEE Trans. Power Delivery, Vol.14, No.3, pp.911-916 (1999)

( 6 ) J. He, G. Yu, R. Zeng, J. Yuan, B. Zhang, J. Zou, and Z. Guan: "Decreasing grounding resistance of substation by deep-ground-well method", IEEE Trans. Power Delivery, Vol.20, No.2, pp.738-744 (2005-4)

( 7 ) R. Zeng, J. He, J. Hu, G. Lu, and B. Luo: "The theory and implementation of corrosion diagnosis for grounding system", Conference Record of the IEEE 37th IAS Annual Meeting-Industry Applications Conference (2002-10)

( 8 ) R. Zeng, J. He, Y. Gao, J. Zou, and Z. Guan: "Grounding resistance measurement analysis of grounding system in vertical-layered soil", IEEE Trans. Power Delivery, Vol.19, No.4, pp.1553-1559 (2004-10)

( 9 ) B. Zhang, R. Zeng, J. He, W. Sun, and J. Yao: "Novel measurement system for grounding impedance of substations and power plants", International Conference on Power System (2000-12)

(10) R. Zeng, J. He, Y. Tu, J. Lee, S. Chang, Y. Gao, J. Zou, and Z. Guan: "Influence of overhead transmission line on grounding impedance measurement of substation", IEEE Trans. Power Delivery, Vol.20, No.2, pp.1226-1234 (2005-4)
(11) J. He, R. Zeng, Y. Tu, J. Zou, S. Chen, and Z. Guan: "Laboratory investigation of impulse characteristics of transmission tower grounding devices", IEEE Trans. Power Delivery, Vol.18, No.3, pp.994-1001 (2003)

(12) Y. Gao, J. He, J. Zou, R. Zeng, and X. Liang: "Fractal simulation of soil breakdown under lightning current", J. Electrostatics, Vol.61, No.3-4, pp.197-207 (2004)

(13) J. He, Y. Gao, R. Zeng, J. Zou, X. Liang, J. Lee, and S. Chang: "Effective length of counterpoise wire under lightning current", IEEE Trans. Power Delivery, Vol.20, No.2, pp.1585-1591 (2005-4)

(14) Y. Gao: "Research on Mechanism of Soil Breakdown and Transient Characteristics of Grounding Systems", Ph.D. dissertation, Tsinghua University $(2003$

Jinliang He (Non-member) born in Changsha, P.R. China, in 1966.

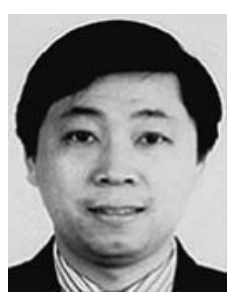
He received his B.Sc. degree from Wuhan University of Hydraulic and Electrical Engineering in Wuhan, China, his M.Sc. degree from Chongqing University in Chongqing, China, and his Ph.D. degree from Tsinghua University in Beijing, China, all in Electrical Engineering, in July 1988, Jan. 1991 and March 1994, respectively. He joined Department of Electrical Engineering, Tsinghua University in Beijing, China in April 1994. He became a professor in the Department of Electrical Engineering, Tsinghua University in Beijing in August 2001. He has been engaged on the study of overvoltages and EMC in power systems and electronic systems, grounding technology, power apparatus, dielectric material, and power distribution automation.

Rong Zeng (Non-member) born in Shaanxi, P.R. China in 1971. He

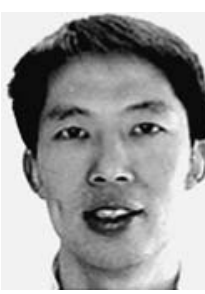
received his B.Sc., M.Eng., and Ph.D. degrees from the Department of Electrical Engineering, Tsinghua University in Beijing, China, respectively in July 1995, July 1997, and July 1999. He joined Department of Electrical Engineering, Tsinghua University in Beijing, China in August 1999. He became an associate professor in the Department of Electrical Engineering, Tsinghua University in Beijing in December 2002. He has been engaged on the study of high voltage technology, grounding technology, power electronics and distribution system automation. 